\title{
Factors determining clinical outcomes in intussusception in the developing world: Experience from Johannesburg, South Africa
}

\author{
C Carapinha, ${ }^{1} \mathrm{MB}$ ChB, FC Paed Surg; M Truter, ${ }^{1} \mathrm{MB}$ BCh; A Bentley, ${ }^{2} \mathrm{PhD}$; A Welthagen; ${ }^{1}$ \\ J Loveland, ${ }^{1}$ MB ChB, FCS (SA), Cert Paed Surg (SA) \\ ${ }^{1}$ Department of Paediatric Surgery, Chris Hani Baragwanath Academic Hospital and Faculty of Health Sciences, University of the \\ Witwatersrand, Johannesburg, South Africa \\ ${ }^{2}$ Department of Family Medicine, Faculty of Health Sciences, University of the Witwatersrand, Johannesburg, South Africa
}

Corresponding author: J Loveland (loveland@wol.co.za)

\begin{abstract}
Background. Rates of open reduction of intussusception were noted to be unacceptably high during an institutional internal audit. Objectives. To determine the impact of revised protocols to better select patients for pneumatic reduction (PR), and document associated morbidity and mortality, and the factors that affect the above.

Methods. Medical records of patients between 3 months and 3 years of age presenting to the Department of Paediatric Surgery at Chris Hani Baragwanath Academic Hospital, Johannesburg, South Africa, from 2007 to 2010 were reviewed. Determining factors, including duration of symptoms, admission C-reactive protein (CRP) level and weight, were analysed against clinical outcomes, notably PR, bowel resection, relook laparotomy and death.

Results. A total of 97 cases were suitable for inclusion. In 62 of these (63.9\%), PR was attempted; this was successful in 32 cases (51.6\%), giving an overall successful PR rate of $33.0 \%$. In 7 of the 62 patients, a pneumoperitoneum was documented during the reduction attempt. Of the 65 patients who underwent surgery, 53 required intestinal resection and 12 had spontaneous or manual reduction. Ileostomy was necessary in 9 patients, and 7 required relook laparotomy. The overall mortality rate was $9.1 \%$. Averages of 'determining factors' assessed against clinical outcome were as follows: mean weight (standard deviation (SD) 7.4 (4.3) kg, mean duration of symptoms (DOS) 3.0 (SD 2.2) days, and admission CRP level $50.9 \mathrm{mg} / \mathrm{L}$ (range 1 - 249.3). Prolonged DOS and a raised CRP level predicted a poor outcome. Conclusions. Despite marked improvements in management and PR outcomes, intussusception remains associated with significant morbidity and mortality. Prolonged DOS and an elevated CRP predict worse outcomes. The use of these markers in association with clinical factors may assist management decisions, specifically with regard to operative or non-operative management. Awareness and education are key to prompt presentation and early diagnosis. Well-defined protocols introduced at all points of contact ensure early recognition and resuscitation as well as prompt referral for definitive management.
\end{abstract}

S Afr Med J 2016;106(2):177-180. DOI:10.7196/SAMJ.2016.v106i2.9672

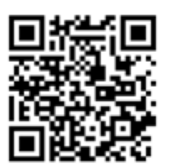

Intussusception is classically divided into idiopathic and pathological causes, with idiopathic intussusception typically occuring between the ages of 6 months and 3 years. ${ }^{[1]}$ Over the age of 3 years, pathological causes predominate, with Meckel's diverticulum, intestinal duplication cysts, polyps and intestinal malignancy the most commonly occurring lead points. ${ }^{[2,3]}$ If intussusception is not rapidly reduced, congestion and subsequent ischaemia of the bowel wall will result, with necrosis, perforation and death as possible outcomes.

Management may be either non-operative or operative. The challenge is in triaging patients correctly into the two management groups, and this is dependent on epidemiological, clinical and radiological factors. Before 2006, an unpublished audit at our institution (Chris Hani Baragwanath Academic Hospital (CHBAH), Johannesburg, South Africa (SA)) demonstrated a subjective reluctance to attempt pneumatic reduction (PR), with the result that the vast majority $(>90 \%)$ of patients were taken directly for laparotomy. Intestinal resection rates were perceived to be high.

In 2006, with the employment of new paediatric surgeons and in co-operation with the Department of Radiology, updated management guidelines were instituted in an attempt to increase the success of PR. These included the introduction of a standardised PR protocol, where both the radiology consultant and the senior paediatric surgery registrar are present during the PR procedure, which is initiated at an inflation pressure of $80 \mathrm{mmHg}$, maintained for 3 minutes, then increased by $20 \mathrm{mmHg}$ every 3 minutes to a maximum of $120 \mathrm{mmHg}$. The PR procedure is performed in the radiology suite without sedation. Failure of PR after this protocol mandated open surgical exploration, and the cohort of patients in this series were not offered a second PR attempt. Absolute contraindications to PR included peritonitis, free intraperitoneal air, established obstruction, haemodynamic instability and multiorgan failure. We simultaneously instituted a prospective observational study to assess our cohort of patients presenting with intussusception and document the impact of our updated guidelines. A further aim was to assess which local epidemiological and clinical factors affected morbidity and mortality, expressed as defined clinical outcomes.

\section{Methods}

After approval by the Human Research Ethics Commitee of the University of the Witwatersrand, Johannesburg (Protocol No. M080206), a prospective observational study of paediatric patients presenting with intussusception to $\mathrm{CHBAH}$ and Charlotte Maxeke Johannesburg Academic Hospital (CMJAH) from January 2007 to April 2010 was undertaken. CHBAH and CMJAH are tertiary/ 
quaternary hospitals affiliated to the University of the Witwatersrand, each having a dedicated paediatric surgical unit. Only data on patients between 3 months and 3 years of age were used. Proven cases of colocolic intussusception were excluded from this study, as these are traditionally thought to occur secondary to a pathological lead point, mandating resection in our unit. Two further cases of suspected intussusception were excluded, as these children died on arrival in the emergency department and did not come to either arm of intervention. Once suitable study candidates had been identified and appropriate consent obtained from their caregiver, a blood sample for C-reactive protein (CRP) was drawn, this being the only extra variable added to the study. CRP was measured by latex immunoassay. Thereafter routine clinical management was instituted as per protocol. At the end of the study period, records of documented cases were reviewed for age, gender, weight, duration of symptoms (DOS), CRP levels, presence of palpable intussusceptum on rectal examination, serology results, radiological investigations including $\mathrm{PR}$, surgical procedure and findings, and histological reports.

The following 'determining factors' were assessed against clinical outcome: DOS, admission CRP level, weight, and presence of palpable intussusceptum per rectum. All pathology specimens from patients who underwent resection were histologically assessed for the presence of transmural necrosis of the intestinal wall. Subgroup analysis was undertaken on patients presenting primarily to CMJAH and CHBAH (referred to as 'in-house' patients) and those transferred from peripheral referral hospitals.

Groups were compared with the MannWhitney $U$-test and independent samples $t$-test for numerical variables, and the $\chi^{2} /$ Fisher's exact tests for categorical variables. Correlation between CRP levels and DOS was assessed using the Pearson's productmoment correlation coefficient. Univariate logistic regression was used to quantify the association between determining factors and clinical outcomes. The 5\% significance level was used. Statistica (Statsoft Inc., USA) software was employed for statistical analysis.

\section{Results}

A total of 108 cases of pediatric intussusception were documented, of which 11 were excluded. Eight patients were aged $>3$ years and underwent primary surgical intervention as per local protocol, and one infant had an exploratory laparotomy for an unknown cause of intestinal obstruction where a jejunojejunal intussusception was discovered. Two children with presumed ileocolic intussusception died on arrival before intervention, and were also excluded. A total of 97 cases of presumed ileocolic intussusception were therefore included in the study.

PR was attempted in 62 patients (63.9\%); it was successful in 32 (51.6\%), equating to an overall success rate of $33.0 \%(32 / 97)$. In 7 cases a pneumoperitoneum was documented during PR. At operation all of these patients were found to have transmural necrosis at the site of perforation, confirming that the perforations were pre-existing and secondary to the advanced state of pathology, as opposed to over-enthusiastic reduction techniques. A total of 65 patients underwent surgical management, 35 primarily and 30 after failed PR. The indication for primary surgery in the 35 cases was established intestinal obstruction with significant abdominal distension, where additional distention of the gastrointestinal tract would cause further respiratory distress. None of the patients who were treated surgically presented with peritonitis. Fifty-three (81.5\%) required intestinal resection. No lead-point pathology was documented histologically. Of the remaining 12 intussusceptions (18.5\%), 7 were found to have reduced spontaneously, all in the PR group. Manual reduction was required in the remaining 5 . Diversion of the gastrointestinal tract by means of ileostomy was necessary in 9 of the 65 patients who required exploration (13.8\%), all in the group that needed resection (Fig. 1).

Averages of the determining factors were as follows: mean (standard deviation (SD)) weight 7.4 (SD 4.3) $\mathrm{kg}$, mean DOS 3.0 (SD 2.2) days, palpable rectal intussusceptum $22 \%$ (21/97), and admission CRP level $50.9 \mathrm{mg} / \mathrm{L}$ (range 1 - 249.3). In the subgroup analysis comparing patients admitted directly to CMJAH and CHBAH with those referred from peripheral hospitals, no significant differences were noted in DOS, admission CRP or weight. There was also no significant difference between the referred group and the in-house group with regard to the rate of successful PR (13/32 (40.6\%) and $18 / 30(60.0 \%)$, respectively) $(p=0.16)$. The mortality rate was nearly three times higher in the referred group (7/55 (12.7\%) v. $2 / 42(4.7 \%)$ in the in-house group), but the difference was not statistically significant $(p=0.18)$. In the subgroup analysis of patients who underwent intestinal resection, CRP levels were compared between two groups: cases in which histological examination confirmed the presence of transmural necrosis (mean CRP $108 \mathrm{mg} / \mathrm{L}$, range $11.8-229.9$ ), and those in which

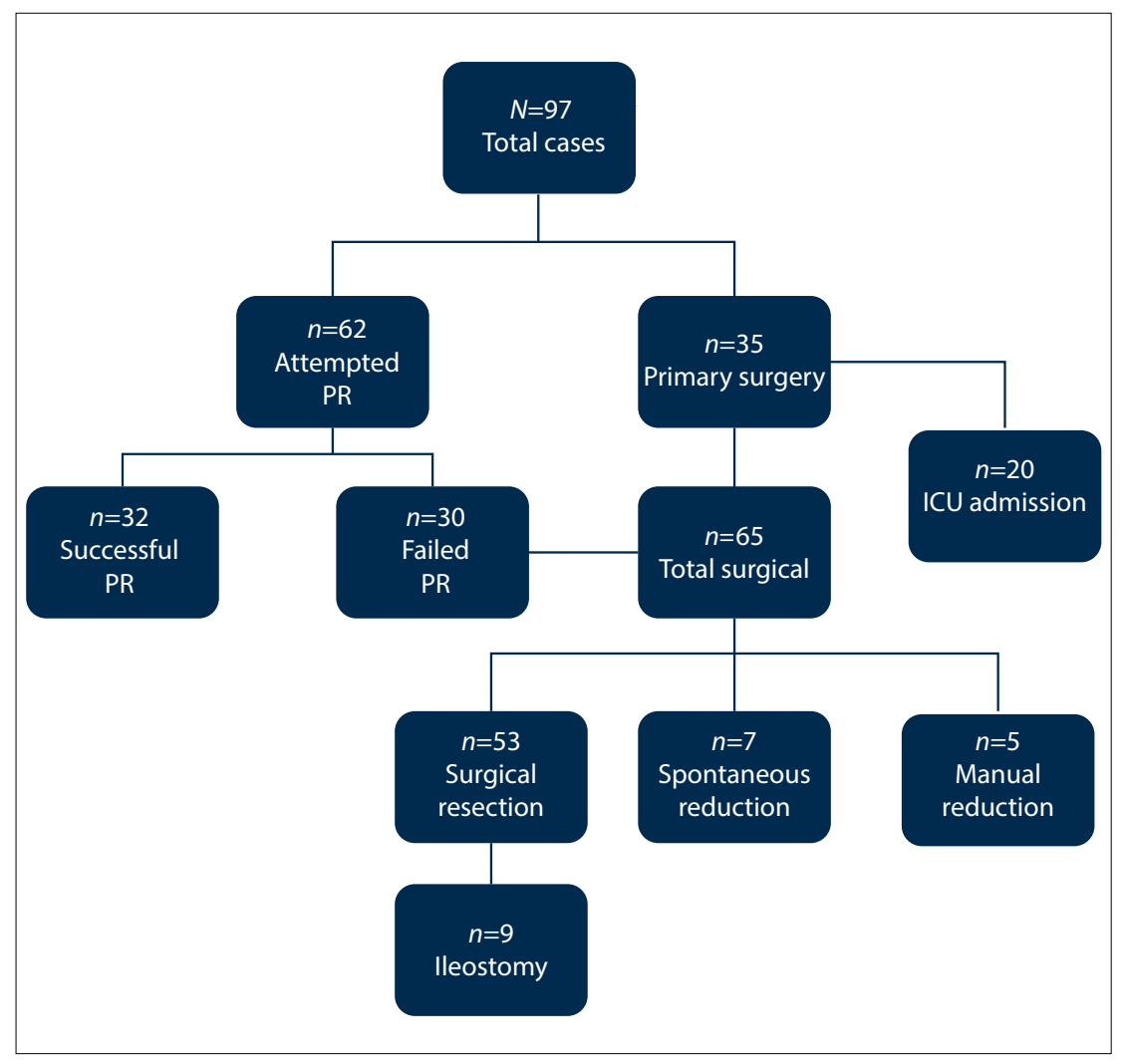

Fig. 1. Flow diagram of intussusception management outcomes. (ICU = intensive care unit.) 


\begin{tabular}{|c|c|c|c|}
\hline Clinical outcomes & Variables & $p$-value & OR $(95 \% \mathrm{CI})$ \\
\hline \multirow[t]{2}{*}{ Failed pneumatic reduction } & DOS (days) & 0.013 & $2.0(1.2-3.5)$ \\
\hline & $\mathrm{CRP}(\mathrm{mg} / \mathrm{L})$ & 0.043 & $1.01(1.001-1.03)$ \\
\hline \multirow[t]{2}{*}{ Relook } & DOS (days) & 0.005 & $2.5(1.3-4.7)$ \\
\hline & $\mathrm{CRP}(\mathrm{mg} / \mathrm{L})$ & 0.025 & $1.01(1.001-1.03)$ \\
\hline Mortality & DOS (days) & 0.020 & $1.4(1.06-1.85)$ \\
\hline
\end{tabular}

transmural necrosis was not found (mean CRP $39.2 \mathrm{mg} / \mathrm{L}$, range $6-245.4)$. The difference was not statistically significant $(p=0.057)$. The relationships between the outcomes and DOS and CRP levels are presented in Table 1. The DOS positively predicted a poorer outcome. The positive correlation between CRP levels and DOS was significant. Seven patients required a relook laparotomy after their initial procedure, the most common indication being abdominal compartment syndrome (ACS) $(n=3)$. Sheath dehiscence occurred in 2 cases and an anastomotic leak after right hemicolectomy and ileocolic anastomosis in 1 case. This last patient required repair after developing a jejunal perforation arising from a serosal tear sustained at initial laparotomy. The mortality rate was $7.2 \%$ (7/97) in the study group, but including the 2 patients who were excluded from the study because they died prior to intervention, this increased to $9.1 \%$. The first of these, a 4-monthold infant with AIDS, died of systemic sepsis secondary to Pneumocystis jirvoveci pneumonia, and the second, a 3-monthold with acute lymphoblastic leukaemia, developed tumour lysis syndrome and acute renal failure. We considered it pertinent to include these in our mortality statistics, as they reflect the overall mortality in patients with intussusception presenting to our unit. Five patients died postoperatively secondary to nosocomial sepsis and multiple organ dysfunction, one infant died from severe gastroenteritis and septic shock a week after hospital discharge, and another developed seizures of unknown aetiology and presented to the emergency department dead on arrival, also in the week following discharge. Univariate logistic regression per clinical outcome is shown in Table 1.

\section{Discussion}

Idiopathic intussusception is common in children, with described rates of successful non-operative reduction of $>90 \%$ and overall mortality approaching zero. ${ }^{[4,5]}$ The present series, as well as others from developing countries, reports associations with significant morbidity and mortality, and emphasises that intussusception should not be underestimated. The last reported experience from CMJAH, by Rogers et al. ${ }^{[6]}$ in 2007, documented a $25 \%$ success rate of $\mathrm{PR}$ in comparison with the current rate of $33 \%$. ${ }^{[6]}$ At the time of the previous study, standardised PR techniques were not in place and treatment was primarily surgical, with a low threshold for opting for resection. The improvement can be explained by the introduction and implementation of standardised protocols in 2006, including guidelines for resuscitation as well as selection for, and performance of, PR. Greater experience in both paediatric surgery and radiology have also had a positive effect.

Our intestinal resection rate of $81.5 \%$ in patients undergoing exploration is high compared with other series. Mayell ${ }^{[7]}$ reported a $22.7 \%$ resection rate, with the rest of the operative cases undergoing spontaneous or manual reduction, possibly demonstrating poor selection for operation in their series. There has been a steady decrease in the number of manual reductions over the years, with our experience demonstrating the lowest manual reduction rate in $\mathrm{SA},{ }^{[6-11]}$ suggesting that institutional protocols for $\mathrm{PR}$ are stringent and reliable.

The average DOS before presentation was 3.0 (SD 2.2) days in our series, which is similar to other series from developing countries. However, this compares poorly with data from the developed world, where the majority of patients have had mere hours of symptoms before presentation. The average DOS for a successful pneumatic reduction in our series is 1.9 (SD 1.3) days, which suggests that stringent protocols excluding attempts at PR in cases with symptoms longer than 24 hours are not well founded. A good history of the exact time of onset of symptoms is important, however, as the longer the DOS, the more physiologically unwell the patient will be. This is confirmed by the predictive nature of DOS for relook laparotomy and mortality, with odds ratios (ORs) of 2.4 and 1.4 , respectively. It is not clear why the DOS does not have any influence on the resection rate in our series, as it would be expected that the longer the duration of a non-reduced intussusception, the greater the chance that ischaemia and necrosis would develop, necessitating bowel resection at laparotomy. It could be postulated that although DOS does not impact on the development of ischaemia and necrosis, it does provide a prolonged opportunity for stasis and bacterial translocation, resulting in the systemic inflammatory response syndrome and multiple organ dysfunction syndrome. Prolonged DOS and intestinal obstruction would also cause excessive fluid losses, causing significant metabolic and electrolyte abnormalities. While intussusception may result in localised intra-abdominal complications, specifically bowel necrosis and perforation, it is the systemic effects, particularly intravascular depletion and metabolic acidosis, that are most concerning and need to be rapidly and aggressively addressed before definitive management.

It is of interest that the DOS and CRP predict for similar clinical outcomes and a positive correlation exists between DOS and CRP, with a Pearson correlation coefficient of 0.4. Although not linear, CRP values increase incrementally the longer the DOS. The exact relationship between these two factors cannot be concluded from this study. However, it is important to clarify that prolonged DOS results in a poor outcome, while a raised CRP level is a marker of, but not contributory to, poor outcome. A raised CRP is predictive of failure of $\mathrm{PR}$ and relook laparotomy, with ORs of 1.01 $(p=0.043)$ and $1.01 \quad(p=0.025)$, respectively. This discrepancy between OR and $p$-value is attributed to the fact that a wide range in CRP values $(1-243.2)$ was analysed. Our results concur with previous studies indicating an association of failed PR with a raised CRP. This study did not evaluate at what level a raised CRP would positively predict failure. However, the converse should hold true: PR in a patient with a normal CRP value on presentation should prove successful in the majority of cases. As positive predictors of outcome, these two factors in combination with other clinical and radiological findings can assist the clinician in stratifying patients for operative and non-operative management.

Relook laparotomies are a described but rare procedure in paediatric surgery. However, in this series a relook laparotomy was required in $10.8 \%$ of all the operative cases, for the reasons listed above. Relook 
laparotomy predicts for mortality with an OR of 44. On closer analysis of the cases in which relook procedures were done, we suspect a common denominator: perioperative fluid resuscitation, particularly in the face of multiorgan dysfunction and postoperative sepsis, may contribute to a significantly positive overall fluid balance, which consequently results in increased third-space fluid, generalised oedema, poor pulmonary compliance, secondary ACS, and poor anastomotic healing. ACS is an under-recognised phenomenon in infants and children.

When comparing in-house patients with those referred from district-level hospitals, CRP levels and DOS were similar. Although no statistical significance was reached, the mortality rate for the referred group was three times higher than that for the in-house group. We attribute this to delayed diagnosis and under-resuscitation at the referring facilities, as well as prolonged transportation times with under-resuscitation during transfer. While this is purely speculative, it does highlight the importance of resuscitation at the point of first contact.

Our mortality rate was high, and comparison of mortality rates can be misleading, with many unaccounted-for factors contributing to death. If we exclude the two patients who died after discharge, our mortality rate would be $7 \%$, comparable to other SA case series. However, our results are not favourable when compared with international series, which document success rates of non-operative reduction in excess of $90 \%$ and mortality rates approaching zero. ${ }^{[3,4]}$ A betterdeveloped health infrastructure, well-informed patient population, better socioeconomic status, an increased ratio of specialists (paediatric surgeons and radiologists) to population and easier access to healthcare all contribute to this significant difference in outcome.

\section{Conclusions}

Intussusception is not a benign condition; on the contrary, it is associated with significant morbidity and mortality. A prolonged
DOS, invariably due to socioeconomic, infrastructural and health management factors, and a raised CRP level are predictors for outcomes in intussusception. The use of DOS and CRP in association with other clinical factors may assist clinicians in making management decisions, specifically regarding operative or nonoperative management. Introduction of PR guidelines has improved our use of this modality, directly contributing to an increase of successful PR from $25 \%$ to $33 \%$. Public awareness and education would result in more prompt presentation to healthcare facilities. Well-defined protocols introduced at all points of contact in the healthcare delivery system would ensure early recognition of intussuception and aggressive resuscitation, followed by appropriate referral for definitive management.

\section{References}

1. Romeo C, Ignacio JR. Intussusception. In: Holcomb GW, Murphy JP, eds. Ashcrafts Pediatric Surgery th ed. Philadelphia: Saunders, 2010:508-516.

2. Maazoun K, Mekki M, Sahnoun L, et al. Intussusception owing to pathologic lead points in children: Report of 27 cases. Arch Pediatr 2007;14(1):4-9. [http://dx.doi.org/10.1016/j.arcped.2006.09.027]

3. Lai WP, Yang YJ, Cheng CN, et al. Clinico-pathological features of intussusceptions in children beyond 3. Lai WP, Yang YJ, Cheng CN, et al. Clinico-pathological fe
five years old. Acta Paediatr Taiwan 2007;48(5):267-271.

4. Parashar UM, Holman RC, Cummings KC, et al. Trends in intussusception-associated hospitalizations and deaths among US infants. Pediatrics 2000;106(6):1413-1421. [http://dx.doi.org/10.1542/ peds.106.6.1413]

5. Jenke AC, Klaassen-Mielke R, Zilbauer M, et al. Intussusception: Incidence and treatment - insights from the Nationwide German Surveillance. J Pediatr Gastroenterol Nutr 2011;52(4):446-451. [http:// dx.doi.org/10.1097/MPG.0b013e31820elbec

6. Rogers TN, Eastman L, Brauer B, et al. Intussusception: Still a surgical disease in Africa. Trop Doct 2007;37(4):254-256. [http://dx.doi.org/10.1258/004947507782333035]

7. Mayell MJ. Intussusception in infancy and childhood in southern Africa: A review of 223 cases. Arch Dis Child 1972;47(251):20-25. [http://dx.doi.org/10.1136/adc.47.251.20]

8. Grant HW, Buccimazza I, Hadley GP. A comparison of colo-colic and ileo-colic intussusception. J Pediatr Surg 1996;31(12):1607-1610. [http://dx.doi.org/10.1016/S0022-3468(96)90031-7]

9. Isdale JM, Saunders WC. Intussusception in Johannesburg: A review of 81 cases. S Afr Med J 9. Isdale JM, Saunders
1986;69(10):610-611.

10. Postma MH, Hadley GP. Intussusception in black children. S Afr Med J 1985;68(6):405-406.

10. Postma MH, Hadley GP. Intussusception in black children. S Afr Med J 1985;68(6):405-406.
11. Wiersma R, Hadley GP. Minimizing surgery in complicated intussusception in the Third World. Wiersma R, Hadley GP. Minimizing surgery in complicated intussusception in the
Pediatr Surg Int 2004;20(3):215-217. [http://dx.doi.org/10.1007/s00383-003-1099-x]

Accepted 16 November 2015 